\title{
On the vapour pressure of radon
}

\author{
A.G.M. Ferreira, L.Q. Lobo * \\ Departamento de Engenharia Química, Universidade de Coimbra, Coimbra 3030-290, Portugal \\ Received 1 March 2007; accepted 23 March 2007 \\ Available online 5 April 2007
}

Dedicated to Professor J. Simões Redinha on the occasion of his 80th birthday

\begin{abstract}
The few, rather scarce, and old data on the vapour pressure and sublimation pressure of radon are re-examined in light of the Wagner equation, which is applied for the first time (as far as we are aware) to the sublimation curve. Numerical coefficients have been established for the representation of both equilibrium curves, and estimates are advanced for the coordinates of the critical point, the triple-point, and the normal boiling temperature of radon. Some important thermodynamic properties, e.g., the enthalpies of vaporisation, sublimation, and fusion are derived, and a $(p, T)$ phase diagram is sketched.
\end{abstract}

(C) 2007 Elsevier Ltd. All rights reserved.

Keywords: Radon; Vapour pressure; Sublimation; Triple-point; Normal boiling temperature

\section{Introduction}

Radon is the heaviest member of Group 0 of the elements. It has been the object of intensive research in the last decades owing to the role it plays in environmental issues for its radioactive nature. In spite of the importance of the rare gases in Physical Chemistry, theoretically and otherwise, the experimental information on the thermodynamic and physical properties of radon is rather scarce. This situation certainly derives from the radioactive quality of the substance which makes it difficult to handle; also, it is commercially unavailable. Probably because of these two facts, the few experimental data registered in the literature come from measurements made in the first years after the discovery of radon in 1900 by F.E. Dorn. These experiments were carried out using small or very small samples of the gas, usually of purity not guaranteed and using techniques now outdated, despite the care with which they were performed and reported. For the phase equilibria concerned, it has been recognized [1] that the measurements

\footnotetext{
${ }^{*}$ Corresponding author. Tel.: +351 239798 733; fax: +351 239798703 .

E-mail address: lqlobo@eq.uc.pt (L.Q. Lobo).
}

made on the vapour pressure of liquid radon by Gray and Ramsay [2] are still the most extensive and reliable ones. As far as we are aware no other set of careful experimental values of this property has been reported in recent times. However, some results were published on the sublimation pressure of (solid) radon but, unfortunately, the values obtained as a function of temperature by Wertenstein [3] were reported in graphical form and the equation given in the original text leads to unacceptably high equilibrium pressures. On the contrary, the measurements made by Kovarik [4] and the equation given by him for the sublimation pressure are too low. Both Wertenstein's and Kovarik's experiments were carried out, respectively, on samples of doubtful purity, and on very small amounts of substance. In this context, only the smooth values reported by Stull [5] at regular round pressures are left, although their experimental origin had not been clearly stated. The results obtained by Pollack [6] from an analysis based on the principle of corresponding states are of the same order of magnitude as those reported by Stull. And the same can be said for the tabulations registered in other sources $[7,8]$. Confronting all these data, it is evident that they do not compare favourably with each other. The coordinates of the critical point and of the normal boiling 
temperature of radon given in the literature also originate from a few old experimental results. Their respective values taken from Ambrose [9] are: $T_{\mathrm{c}}=377 \mathrm{~K} ; p_{\mathrm{c}}=6280 \mathrm{kPa}$; and $T_{\mathrm{b}}=211.4 \mathrm{~K}$, although alternative figures can be found in other sources. An estimate of the triple-point temperature has been advanced [6]: $T_{\mathrm{tr}}=202 \mathrm{~K}$. The value of the corresponding triple-point pressure $p_{\mathrm{tr}}=66.7 \mathrm{kPa}$ registered in the same reference is only tentative and it was not considered in our review article on the triple-point of low-melting substances [10].

While better experimental values for the vaporisation and sublimation equilibrium pressures of radon are not available, in this work we re-examine the existing information by using the well established Wagner equation [11], which we believe is the most capable and powerful analytical instrument in dealing with vapour pressure data. Values of some derived thermodynamic quantities (e.g. the enthalpies of vaporisation and sublimation) are also presented here.

\section{Results and discussion}

The vapour pressure equation proposed by Wagner in $1973[11]$ is

$\ln p=\ln p_{\mathrm{c}}+\left(T_{\mathrm{c}} / T\right)\left(a_{1} \tau+a_{2} \tau^{1.5}+a_{3} \tau^{n}+a_{4} \tau^{m}\right)$,

where $p$ is the equilibrium vapour pressure, $T$ is the temperature, subscript $\mathrm{c}$ refers to the critical point, the $a_{i}$ are adjustable parameters, and $\tau=1-T_{\mathrm{r}}$ with $T_{\mathrm{r}}=T / T_{\mathrm{c}}$. In the original version of equation (1), the exponents were taken as $n=3$ and $m=6$ but other values of these and the other exponents have been used in more recent works. Usually $m=2 n$. In particular, it has been suggested [12], and indeed said to be internationally accepted [13], that by making $m=5$ equation (1) leads to the best form of the Wagner equation, both in adjusting to experimental data and in extrapolation. One additional advantage of equation (1) is that it can be used to estimate reliable values of the critical pressure if enough vapour pressure measurements have been made on the substance under examination. In other words, in the Wagner equation, $p_{\mathrm{c}}$ can be treated as one further adjustable parameter as well as the four parameters $a_{i}$ [14-16].

Gray and Ramsay [2] carried out two series of experiments: one of 28 measurements of the vapour pressure of radon spanning temperatures from (273 to 375 ) K; and a second one of 14 points encompassing the interval from (263 to 377.5$) \mathrm{K}$. In the light of present standards, these values of the measured pressure are somewhat scattered and their temperature scale is difficult to assess. By using both sets of values and the critical temperature measured by Gray and Ramsay, which in our assessment should be $T_{\mathrm{c}}=377.7 \mathrm{~K}$ in ITS-90, equation (1) with $n=2.5$ and $m=5$ leads to $a_{1}=-5.1737, a_{2}=1.4220, a_{3}=-5.1052$, $a_{4}=12.3409$, and to an estimate of $p_{\mathrm{c}}=(6189 \pm 168) \mathrm{kPa}$. This value of the critical pressure compares much favourably with that generally selected by others $[6,9]$. The same is true for the boiling temperature, which in the literature [9] is given as $T_{\mathrm{b}}=211.4 \mathrm{~K}$ whereas we obtain $T_{\mathrm{b}}=$ $211.9 \mathrm{~K}$. The standard deviation of this fitting is $\sigma(\ln p)= \pm 0.0380$.

To deal with the sublimation pressure curve, it is commonly accepted that much simpler equations are sufficient, namely that of $\ln p$ as a function of $T^{-1}$ and the Kirchhoff equation. Both these forms have been proposed for radon $[3,6]$. Pollack arrived at one such equation by applying the principle of corresponding states to rare gas solids [6]. Not long ago, we have shown [17] that a more complete equation for the sublimation curve can be established. The application of this expression needs some ancillary data, especially on the heat capacities and molar volumes of the equilibrium solid and vapour phases which are not available in the case of radon. With the exception of the equation proposed by Pollack, the sublimation curves obtained for radon from the equations recorded in the literature do not cross the vapour pressure curve obtained by us. The crossing point obtained from Pollack's equation occurs at $T=191 \mathrm{~K}$ and $p=38.0 \mathrm{kPa}$, which lie far apart from those that have been suggested in the literature. This situation led us to examine the possibility of describing the sublimation curve of radon by using an equation of the same form as that established by Wagner. Replacing the critical point coordinates $\left(T_{\mathrm{c}}, p_{\mathrm{c}}\right)$ in equation (1) for those of the triple-point $\left(T_{\mathrm{tr}}, p_{\mathrm{tr}}\right)$ one obtains:

$\ln p=\ln p_{\text {tr }}+\left(T_{\text {tr }} / T\right)\left(a_{1}^{\prime} \theta+a_{2}^{\prime} \theta^{1.5}+a_{3}^{\prime} \theta^{n}+a_{4}^{\prime} \theta^{m}\right)$

where $\theta=1-\left(T / T_{\mathrm{tr}}\right)$. This assumption seems plausible for three main reasons: firstly, because the trend and form of the sublimation curve are not too much different from those of its vaporisation counterpart, especially not far from the triple-point; secondly, because of the flexible form of the Wagner equation itself; and, finally, because in the limit of zero temperature equation (2) leads to $p=0$ provided the condition $\sum a_{i}^{\prime}<0$ is enforced. In the absence of measured values of the triple-point coordinates equation (2) cannot be used directly. However, we can arrive at reliable estimates of the unknown values by iteration starting with an approximate guess for the triple-point temperature $T_{\mathrm{tr}}$ and having in mind that equations (1) and (2) should give the same figure for $p_{\text {tr }}$, since the triple-point is common to both curves. From the values compiled by Stull [5], using this procedure in conjunction with the vapour pressure equation (1) with the $a_{i}$ parameters noted above (and a reasonable estimate of $T_{\mathrm{tr}}$ ), we arrive at converging values of the triple-point coordinates: $T_{\mathrm{tr}}=(200.0 \pm 0.1) \mathrm{K}$ and $p_{\mathrm{tr}}=(58.8 \pm 0.6) \mathrm{kPa}$. The $a_{i}^{\prime}$ parameters obtained for equation (2) are: $a_{1}^{\prime}=-10.3220 ; a_{2}^{\prime}=1.8802 ; a_{3}^{\prime}=$ -7.4723 ; and $a_{4}^{\prime}=-15.3580$. The standard deviation for the sublimation pressure obtained from this fitting is $\sigma(\ln p)= \pm 0.0068$.

Having established reliable expressions for the vapour pressure curve, equation (1), and for the sublimation curve, equation (2), one can extract immediately from them by using Clapeyron equation plausible values of the molar 


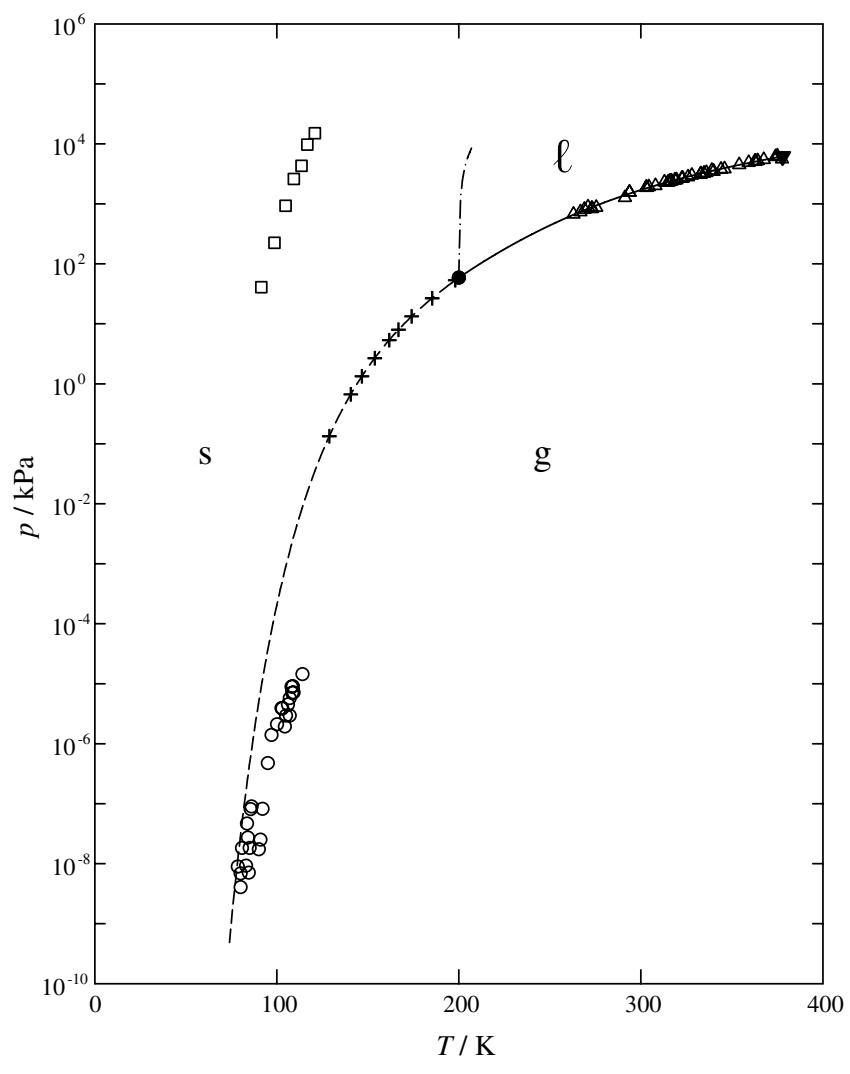

FIGURE 1. Plot of the pressure against temperature to show the phase diagram of radon. The points are experimental: $(\triangle)$ reference [2]; $(\square)$ reference [3]; $(\bigcirc)$ reference [4]; $(+)$ reference [5]. The curves are calculated: $(-)$ from equation (1); (---) from equation (2); (--) from equation (3). The dot ( ) indicates the triple-point, and the inverted triangle $(\boldsymbol{\nabla})$ is the critical point. s, 1 , and $\mathrm{g}$ are the solid, liquid and gas phases, respectively.

enthalpies of vaporisation $\Delta_{1}^{\mathrm{g}} H_{\mathrm{m}}$ and sublimation $\Delta_{\mathrm{cr}}^{\mathrm{g}} H_{\mathrm{m}}$, at least near the triple-point. The estimates for these two important quantities obtained in this manner at the triplepoint temperature are: $\Delta_{1}^{\mathrm{g}} H_{\mathrm{m}}=15.7 \mathrm{~kJ} \cdot \mathrm{mol}^{-1}$ and $\Delta_{\mathrm{cr}}^{\mathrm{g}} H_{\mathrm{m}}=17.2 \mathrm{~kJ} \cdot \mathrm{mol}^{-1}$. These are to be compared with (17.8 and 19.2$) \mathrm{kJ} \cdot \mathrm{mol}^{-1}$, respectively, given in Pascal's treatise [18]. At the triple-point, the molar enthalpy of fusion $\Delta_{\mathrm{cr}}^{1} H_{\mathrm{m}}$ is obtained from the two thermodynamic quantities given just above since $\Delta_{\mathrm{cr}}^{1} H_{\mathrm{m}}=\Delta_{\mathrm{cr}}^{\mathrm{g}} H_{\mathrm{m}}-\Delta_{1}^{\mathrm{g}} H_{\mathrm{m}}$. The resulting value is $\Delta_{\mathrm{cr}}^{1} H_{\mathrm{m}}=1.4 \mathrm{~kJ} \cdot \mathrm{mol}^{-1}$. Of course, from the enthalpy of fusion one can try and obtain an estimate of the slope of the fusion curve at the triple-point provided a necessary value of the change in the molar volume $\Delta_{\mathrm{cr}}^{1} V_{\mathrm{m}}$ at $T=T_{\text {tr }}$ is available, as is imposed by Clapeyron equation:

$\mathrm{d} p / \mathrm{d} T=\Delta_{\mathrm{cr}}^{1} H_{\mathrm{m}} /\left(T \cdot \Delta_{\mathrm{cr}}^{1} V_{\mathrm{m}}\right)$, where $p$ is the pressure of fusion. However, there are no measured values of the molar volumes of solid and liquid radon at the triple-point. Rough figures of the densities of both phases are indicated in Pascal's book [18], but no mention is given to the sources of this information or to the temperatures to which they refer. Assuming that such densities are relative to that of water at $T=288 \mathrm{~K}$ and that the molar volumes of solid and liquid radon at the triplepoint are not significantly different from those, it is easy to extract from them, by the Clapeyron equation plausible values of the slope of the fusion curve, $\mathrm{d} p / \mathrm{d} T=$ $1.2 \mathrm{MPa} \cdot \mathrm{K}^{-1}$. The sketch of a tentative phase diagram of radon is indicated in figure 1 .

In summary, we may conclude that: (i) for the sublimation curve an equation similar to that established by Wagner for vaporisation can be used with satisfactory results; and (ii) reasonable (better, we believe) values for the coordinates of the critical point, the triple-point, and the normal boiling temperature of radon have been obtained, as well as values for the enthalpies of transition between the solid, liquid and gaseous phases at the triple-point.

\section{References}

[1] L. Stein, in: C.A. Hampel (Ed.), The Encyclopedia of the Chemical Elements, Reinhold, New York, 1968.

[2] R.W. Gray, W. Ramsay, J. Chem. Soc., Trans. 95 (1909) 1073-1085.

[3] L. Wertenstein, Proc. Roy. Soc. Lond. A 150 (1935) 395-410.

[4] A.F. Kovarik, Phil. Mag. VII (1927) 1262-1275.

[5] D.R. Stull, Ind. Eng. Chem. 39 (1947) 540-550.

[6] G.L. Pollack, Phys. Rev. A 2 (1970) 38-42.

[7] CRC Handbook of Chemistry and Physics, 76th ed., CRC Press, New York, 1995.

[8] G.W. Kaye, T.H. Laby, Tables of Physical and Chemical Constants, 15th ed., Longman, London, 1986.

[9] D. Ambrose, NPL Report Chem. 107, Teddington, 1980.

[10] L.A.K. Staveley, L.Q. Lobo, J.C.G. Calado, Cryogenics 21 (1981) 131-144.

[11] W. Wagner, Cryogenics 13 (1973) 470-482.

[12] D. Ambrose, J. Chem. Thermodyn. 18 (1986) 45-51.

[13] W. Wagner, J. Chem. Thermodyn. 26 (1994) 399-413.

[14] C. Henderson, D.G. Lewis, P.C. Prichard, L.A.K. Staveley, I.M.A. Fonseca, L.Q. Lobo, J. Chem. Thermodyn. 18 (1986) 10771088.

[15] A.G. Cubitt, C. Henderson, L.A.K. Staveley, I.M.A. Fonseca, A.G.M. Ferreira, L.Q. Lobo, J. Chem. Thermodyn. 19 (1987) 703710 .

[16] M.H. Streatfeild, C. Henderson, L.A.K. Staveley, A.G.M. Ferreira, I.M.A. Fonseca, L.Q. Lobo, J. Chem. Thermodyn. 19 (1987) 11631171.

[17] L.Q. Lobo, A.G.M. Ferreira, J. Chem. Thermodyn. 33 (2001) $1597-$ 1617.

[18] P. Pascal, Nouveau Traité de Chimie Minérale [I], Masson, Paris, 1956.

JCT $07-82$ 